Results During this period 43 questionnaire were returned. The majority felt the service was already at a very high standard citing phrases, 'caring and reassuring', 'made to feel special'. One family requested more information pertaining to the drugs used during the stabilisation and transfer process and one family felt the team's projected timings could have been more accurate for them to organise their other family responsibilities.

Conclusions This study has been insightful into the parent's experiences of having their child transferred by a retrieval team. Actions are underway to address areas highlighted by families as discussed above.

We plan to replicate this study in other regions to gain a national perspective of parents' perceptions of the transfer process.

\section{A SURVEY OF STRESS RESOURCES AMONG PARENTS OF CRITICALLY ILL CHILDREN IN PICU WARD}

doi:10.1136/archdischild-2012-302724.0035

'M Soltanian, ${ }^{2} \mathrm{~F}$ Nikouee, ' $\mathrm{M}$ Edraki, 'S Montaseri. 'Fatemeh College of Nursing \& Midwifery, Shiraz University of Medical Sciences, Shiraz; ${ }^{2}$ Community Medicine Department, Fasa University of Medical Science, Fasa, Iran

Introduction Pediatric intensive care unit (PICU) has long been recognized as a highly stressful place. Admission of a child to the PICU maybe one of the most stressful events for parents. Therefore this study was done to determine stressor resources of parents in PICU.

Method A descriptive cross sectional study was carried out by using data obtained through "parental stressor scale: (PSS: PICU) to examine sources of stress among parents. A convenience sample of 25 parents whose child had recently admitted to PICU were subjects for study demographic data were collected using an investigator designed questionnaire, then parental stress was measured with parental stressor scale the PSS:PICU scale is a 37 item instrument in 7 subscale that uses a likert-type scale to measure source of stress in PICU.

Findings After measurement of stress in parents with PSS result revealed that in a comparison of mean score on the 7 dimensions of the PSS: PICU parent found the "Alteration in parental role "to be the greatest source of their stress $(3.74 \pm 0.61)$ and the second highest ranking stressor was a "Not knowing what to expect".(3.56 \pm 0.70$)$.

Conclusion Result suggests the need to prepare parents for role alteration when a child is admitted to PICU. They should also be encouraged to participate in the child's care and provides comfort measures. We need to consider creating spaces that not only address family needs to remain present with their child, but also creating space for parents to partner in decision making and care planning.

\section{PATIENT SAFETY: CULTURE SHIFTS OVER TIME}

doi:10.1136/archdischild-2012-302724.0036

J Maaskant, M de Neef, J Wielenga. Women' and Children' Clinic, Academic Medical Center, Amsterdam, The Netherlands

Background and aims Although patient safety is a major topic in organisations worldwide, many patients are still confronted with errors that cause discomfort, harm and sometimes even death. A safety program including activities to improve the healthcare processes and techniques is more effective when combined with a positive safety culture. The purpose of this study was to evaluate the safety culture in order to fine-tune our safety program.

Methods A survey was undertaken among paediatricians and specialized nurses by means of the Hospital Survey on Patient Safety Culture before (2009) and after (2011) the start of a safety program on five paediatric wards. The results of these five wards (group A) were compared with two wards (group B) where the program was implemented before 2009 .
Results In group A significant more positive ratings were found on seven dimensions in 2011 compared to 2009:

- 'frequency of events reporting': $64 \%$ versus $47 \%(p=0.003)$

- 'non punitive response to error': $93 \%$ versus $80 \%(p=0.001)$

- 'communication openness': $97 \%$ versus $87 \%(p=0.001)$

- 'feedback and communication about error': $90 \%$ versus $68 \%$ $(\mathrm{p}=0.000)$

- 'hospital management support for safety': $61 \%$ versus $37 \%$ $(\mathrm{p}=0.000)$

- 'staffing': $50 \%$ versus $32 \%(p=0.002)$

- 'overall perceptions of safety': $64 \%$ versus $50 \%$ ( $p=0.016)$

The differences between group A and B became smaller on all dimensions except 'communication openness'.

Conclusions Implementation of a patient safety programme improves the culture on most dimensions. Our results guide future activities focused on the dimensions that did not reach satisfactory levels.

\section{ASSESSING CLINICIANS' KNOWLEDGE AND CONFIDENCE PERFORMING FOUR EVIDENCE-BASED PRACTICES IN THE NICU USING THE NEONATAL UNIT CLINICIAN ASSESSMENT TOOL}

doi:10.1136/archdischild-2012-302724.0037

'W Higman, 'L Wallace, ${ }^{2} \mathrm{~K}$ Blake, 'S Law, 'B Jackson. 'ARC HLI, Coventry University, ${ }^{2}$ UHCW NHS Trust, Coventry, UK

The evidence that breast milk feeding reduces mortality and short and long-term morbidity among premature and small babies is well established but breastfeeding rates in neonatal units in the UK remain low. We present a case study of how a tertiary hospital unit with 100 staff undertook the Neonatal Unit Clinician Assessment Tool (NUCAT), an on-line objective knowledge test with ratings of confidence and knowledge in breastfeeding, breast milk expression, kangaroo care and positive touch knowledge and practices. Fifty one medical and nursing clinicians completed NUCAT. We report descriptive statistics $(n=51)$, and paired t tests for pre-post knowledge test confidence items, and difference statistics (Chi squared and $t$ tests or one way ANOVAs for establishing differences in knowledge and confidence (Dependent Variables) on personal descriptive variables (Independent Variables).Confidence in knowledge was significantly reduced when individuals received their scores, but confidence in breastfeeding practice was not reduced. More staff scored better on the practical than knowledge based areas. Doctors, those with more neonatal experience and years since qualifying were not more knowledgeable than other clinicians overall, but clinicians with more senior positions knew more about the knowledge underpinning breastfeeding practices. Data reported include regression of job type, prior training, years since qualified, years working in neonatal care, intensity of direct care duties, on knowledge and confidence scores which help to target future training on those with most urgent job requirements for these practice skills. Training recommendations are discussed.

\section{ALICE IN CYBERLAND: USE AND ABUSE OF INTERNET BY YOUNG PEOPLE AND HOW TO RESPOND TO THEM}

doi:10.1136/archdischild-2012-302724.0038

PA Michaud. Multidisciplinary Unit for Adolescent Health, University Hospital CHUV, Lausanne, Switzerland

Children and adolescents living nowadays were born with internet and the so called information and communication technology "ICTs", which belong, in most countries of the world, to their everyday life. Not only are pediatricians and health professionals faced with questions from an increasing number of parents, but they will more and more in the future have to consider the assessment of 